\title{
ISOLATION OF CELLULOSE MICROFIBRILS - AN ENZYMATIC APPROACH
}

\author{
Sreekumar Janardhnan* and Mohini M. Sain
}

\begin{abstract}
Isolation methods and applications of cellulose microfibrils are expanding rapidly due to environmental benefits and specific strength properties, especially in bio-composite science. In this research, we have successfully developed and explored a novel bio-pretreatment for wood fibre that can substantially improve the microfibril yield, in comparison to current techniques used to isolate cellulose microfibrils. Microfibrils currently are isolated in the laboratory through a combination of high shear refining and cryocrushing. A high energy requirement of these procedures is hampering momentum in the direction of microfibril isolation on a sufficiently large scale to suit potential applications. Any attempt to loosen up the microfibrils by either complete or partial destruction of the hydrogen bonds before the mechanical process would be a step forward in the quest for economical isolation of cellulose microfibrils. Bleached kraft pulp was treated with OS1, a fungus isolated from Dutch Elm trees infected with Dutch elm disease, under different treatment conditions. The percentage yield of cellulose microfibrils, based on their diameter, showed a significant shift towards a lower diameter range after the high shear refining, compared to the yield of cellulose microfibrils from untreated fibres. The overall yield of cellulose microfibrils from the treated fibres did not show any sizeable decrease.
\end{abstract}

Keywords: Cellulose, Cellulose microfibrils, Fungal / Enzyme pretreatment, Cellulose microfibrils isolation, Hydrogen bonds

Contact information: Department of Chemical Engineering and Applied Chemistry, 200 College Street University of Toronto, Toronto, ON-M5S 3E5, Canada. "Corresponding author:

s.janardhnan@utoronto.ca

\section{INTRODUCTION}

Cellulose, the most abundant biopolymer on earth, is poly $(\beta-1,4, \mathrm{D}$ anhydroglucopyranose), which through a regular network of inter and intramolecular hydrogen bonds is organized into perfect sterioregular configurations called microfibrils. Each chain is stabilized by intrachain hydrogen bonds formed between the pyranose ring oxygen in one residue and the hydrogen of the $\mathrm{OH}$ group on $\mathrm{C} 3$ in the next residue $(\mathrm{O} 5 . . . \mathrm{H}-\mathrm{O} 3$ ') and between the hydroxyls on $\mathrm{C} 2$ and $\mathrm{C} 6$ in the next residue $(\mathrm{O} 2-\mathrm{H} . . . \mathrm{O} 6$ ') (Liang and Marchessault 1959).

During biosynthesis, cellulose microfibrils are synthesized by the plasma membrane using an enzyme called cellulose synthase and are deposited onto the cell wall. In higher plants, despite its chemical simplicity, the physical and morphological structure of native cellulose is complex and heterogeneous, and in cell walls cellulose molecules are intimately associated with other polysaccharide moieties, resulting in even more 
complex morphologies. Breakdown of these close physical and chemical associations between cellulose and other polysaccharides in a plant cell wall is vital for any economical utilization of these polymers. Researchers have achieved significant progress in converting lignocellulosic materials to materials of engineering importance such as reinforcing fibres, bioplastics and even biofuels.

The elementarization of natural fibres into their elementary cellulosic constituents such as nano- and microfibrils is gaining wider attention due to their (1) high strength and stiffness (Tashiro 1991), (2) high reinforcing potential, and (3) their biodegradability and renewability. Depending on the degree of elementarization, the defects and dimensions of the partly crystalline fibres of wood decrease, thereby improving their strength properties. The literature differentiates between Microfibrillated Cellulose (MFC) obtained through a mechanical homogenization (Herrick et al., 1983) and Microcrystalline Cellulose (MCC) that is generated by chemical treatment of various plant fibres. MFC has an aspect ratio around 50 to 100 and is extensively investigated for its reinforcing potential, while MCC with an aspect ration of about 3 is widely used as rheology control agents and as binders in the pharmaceutical industry.

Preparation and application of nanocomposites using cellulose nano- and microfibrils are expanding rapidly in biocomposite science. Numerous other high-end potential applications for cellulose microfibrils are currently being explored. Poor economics due to a high energy requirement in the isolation of cellulose microfibrils is a key challenge that could hamper the current momentum in the direction of commercialization. Microfibrils have been generated in the laboratory through a combination of high energy refining in a PFI mill, and subsequent cryocrushing under the presence of liquid nitrogen (Chakraborty and Sain 2005).

\section{Isolation of Cellulose Microfibrils}

Microfibrils are joined laterally by means of hydrogen bonding (Brown et al. 1976). In the cited study, as the microfibrils were generated, they were found to coalesce laterally through interfibrillar hydrogen bonding to form bundles. As stated by the authors, "the bundles associate with neighboring bundles to produce a composite ribbon of cellulose microfibrils".

The glucose and cellobiose structures show the presence of several hydroxyl radicals in the cellulose chain, and all these hydroxyl groups participate in hydrogen bonding. The interfibrillar hydrogen bonding energy has to be overcome in order to separate the microfibrils into individual entities. More than one type of H-bond is present in cellulose - intermolecular and intramolecular, so only a range of values can be used to quantify the hydrogen bond strength. This energy (U) for cellulose ranges between 19 and $21 \mathrm{MJ} / \mathrm{kg}$ mol (Nissan et al., 1985).

Young's modulus $(E)$ of a hydrogen bond-dominated solid such as paper has been quantified (Nissan et al. 1985) as follows:

$$
E=<\mathrm{k}_{\mathrm{R}}>n^{1 / 3}
$$


where $R$ is the total $\mathrm{H}$-bond length, $<k_{\mathrm{R}}>$ is the average value of the force constant for stretching $R$ by a unit distance, and $n$ is the effective number of $\mathrm{H}$-bonds per unit volume involved in taking up strain under uniaxial stress conditions.

Microfibrils are more flexible and agglomerate less in the presence of water. Fengel (1974) indicated that intensive disintegration in a homogenizer could split even the elementary fibrils and microfibrils down to molecular diameters.

Any attempt to loosen up the microfibrils by either complete or partial destruction of the hydrogen bonds before the mechanical process would be a step forward in the quest for energy-efficient generation of cellulose microfibrils. The focus of this research is to investigate and establish an enzymatic chemistry that would partially or completely nullify the hydrogen bonds between the microfibrils, making their isolation energyefficient.

\section{Enzyme Technology in Fibre Processing}

The application of enzymes in fibre processing has been mainly directed towards the degradation or modification of hemicelluloses and lignin, while retaining the cellulosic portion. The enzymatic approach in the fibre processing sector has been based the idea of selected hydrolysis of certain components or limited hydrolysis of several components in the fibre. Some of the important areas of applications are (1) Fibrillation, inter-fibre bonding and strength enhancement (Bolaski et al. 1959; Yerkes 1968; Nomura 1985), (2) Drainage (Fuentes, 1988), (3) Modification of pulp properties (Uchimoto 1988; Paice 1984; Senior 1988; Jurasek 1988), (4) Enzymatic pulping (Nazareth 1987; Sharma and 1987, Morvan, C., 1990), and (5) Enzymatic pretreatments for bleaching (Tolan 1992; Viikari 1990).

Although enzymes have been widely used to modify cellulosic fibres for various applications, there hasn't been any research effort to understand and utilize the enzyme fibre interaction at microfibrilar level. An understanding of the chemistry at this level and its exploitation to isolate high strength micro- and nanofibrils from plant cell walls in an economical manner would be a huge step towards isolation of cellulose microfibrils and their commercial scale utilization in various applications.

\section{EXPERIMENTAL}

\section{Materials}

Wood Fibre: Bleached kraft pulp - northern black spruce was used as the starting material for the isolation of microfibrils. Typical composition is described in Table 1.

\begin{tabular}{lc}
\hline Table.1. Composition of Bleached Kraft Pulp \\
\hline & $\%$ \\
Composition & 86 \\
\hline Cellulose & 14 \\
Hemicellulose & \\
\hline
\end{tabular}

Fungus: The fungus OS1, isolated in our laboratory from Elm tree infected with Dutch elm disease was used as the source of enzyme for the fibre treatment. 


\section{Methods}

Bio-treatment: Twenty-four grams of oven-dry bleached kraft fibre was soaked overnight, disintegrated in 2 liters of water, and autoclaved for 20 minutes. A 24 gram sample size of fibre was chosen, as it was the optimum fibre charge to the high shear refiner that was used for further mechanical defibrillation for cellulose microfibrils isolation. OS1 fungal culture was added to this fibre suspension in a sterile flask with appropriate amount of sucrose and yeast extract to support the fungal growth. The fungus was left to act on the fibres at room temperature for different time duration with slow agitation. The fibres were autoclaved after their respective treatment time, washed and made into sheets of $10 \%$ fibre consistency ready for the mechanical refining and cryocrushing.

High shear refining: The fibres at $10 \%$ consistency were then sheared in a refiner for 125000 revolutions.

Cryocrushing: The refined fibres were then subjected to cryocrushing in which the fibres were frozen, using liquid nitrogen, and high shear was applied, using a mortar in a pestle. This step is critical in librating the microfibrils from the cell wall. The cryocrushed fibres were then dispersed in to water suspension using a disintegrator and filtered through a 60-mesh filter. The filtrate, a dilute water suspension of microfibrils, was used for further investigation.

\section{Characterization of Ophiostoma Ulmi treated fibres}

Weight loss: The weight loss of the bio-treated fibres was determined by simple difference between the weight of fibres before and after treatment.

Fibre composition: The cellulose and hemicellulose contents of the fibre after the bio-treatment were determined using the procedure adapted from Zobel and McElwee 1966).

\section{Cellulose Microfibril Characterization}

Scanning electron microscopy (SEM) and transmission electron microscopy (TEM) were used to understand the surface morphology and diameter distribution of the treated fibres and cellulose microfibrils isolated.

\section{RESULTS AND DISCUSSION}

The results presented here focus on the effect of OS1 fungal pretreatment of bleached kraft softwood fibres on yield and diameter distribution of cellulose microfibrils obtained through subsequent defibrillation techniques such as high shear refining and cryocrushing. The action of fungal treatment on the morphology and the capacity of the bio-treatment to facilitate the internal defibrillation are extensively detailed here through Scanning Electron Microscopy (SEM) and Transmission Electron Microscopy (TEM). The impact of bio-treatment and its extent on the physical and chemical characteristics of the fibres were studied by determining weight loss and cellulose content of the fibres. 


\section{Effect of OS1 Fungal Pretreatment of Fibres on Cellulose Microfibril Yield and Fibre Diameter Distribution}

One of the major challenges impeding the isolation of cellulose microfibrils on a sizable scale for any intended application is the predominating hydrogen bonding between the cellulose microfibrils and also between microfibrils and hemicellulose. Cellulose microfibrils are generated and isolated through a combination of high energy refining, and subsequent cryocrushing under the presence of liquid nitrogen. A key reason for high shear refining of the fibres is to cause internal defibrillation, a process where only a minor portion of the total energy supplied to the refiner is utilized for internal defibrillation.

The interfibrillar hydrogen bonding energy has to be overcome in order to separate the microfibres into individual entities. This association energy for cellulose ranges between 19 and $21 \mathrm{MJ} / \mathrm{kg}$.mol, with $20 \mathrm{MJ} / \mathrm{kg} . \mathrm{mol}$ being used as an average value in most cases. If this value is taken to be the intermolecular $\mathrm{H}$-bond energy binding the fibres together, then this much energy should be supplied to separate the microfibres into separate entities.

One of the main reasons to choose OS1 as the first fungal candidate for fibre treatment is our prior knowledge (Modification of interface in natural fibre reinforced composites, MASc Thesis, Deepak Gulati, 2006) of their effect on hemp fibres - its capacity to degrade and probably hydrolyze the cellulose. In this work, bleached kraft pulp was pretreated with OS1 fungus to study its effect on (a) overall yield of microfibrils, (b) number averaged fibre diameter distribution.

\section{Yield of Cellulose Microfibrils}

The yield of cellulose microfibrils is determined as the percent by weight of microfibrils that pass through a 60-mesh screen after refining and cryocrushing. The yield comparison is detailed in the Fig. 1. The overall yield of cellulose microfibrils from OS1 treated fibre is seen to decrease by an average of $5 \%$. The decrease in yield of microfibrils seems to be noticeable only after a minimum of 4 days of treatment, which indicates that the fungus needs a minimum of 4 days to establish an active community and produce enzymes in an effective quantity.

The yield of microfibrils is seen to stabilize after 5 days of treatment, and there is no noticeable decrease with any further extent of treatment. This observation contradicts results of the earlier study of the effect of OS1 fungus on hemp fibres, which showed a significant activity of the fungus towards cellulose accompanied by a significant loss in the fibre strength after 4 days of treatment (Modification of interface in natural fibre reinforced composites, MASc Thesis, Deepak Gulati, Department of Chemical Engineering and Applied Chemistry, University of Toronto, 2006). 


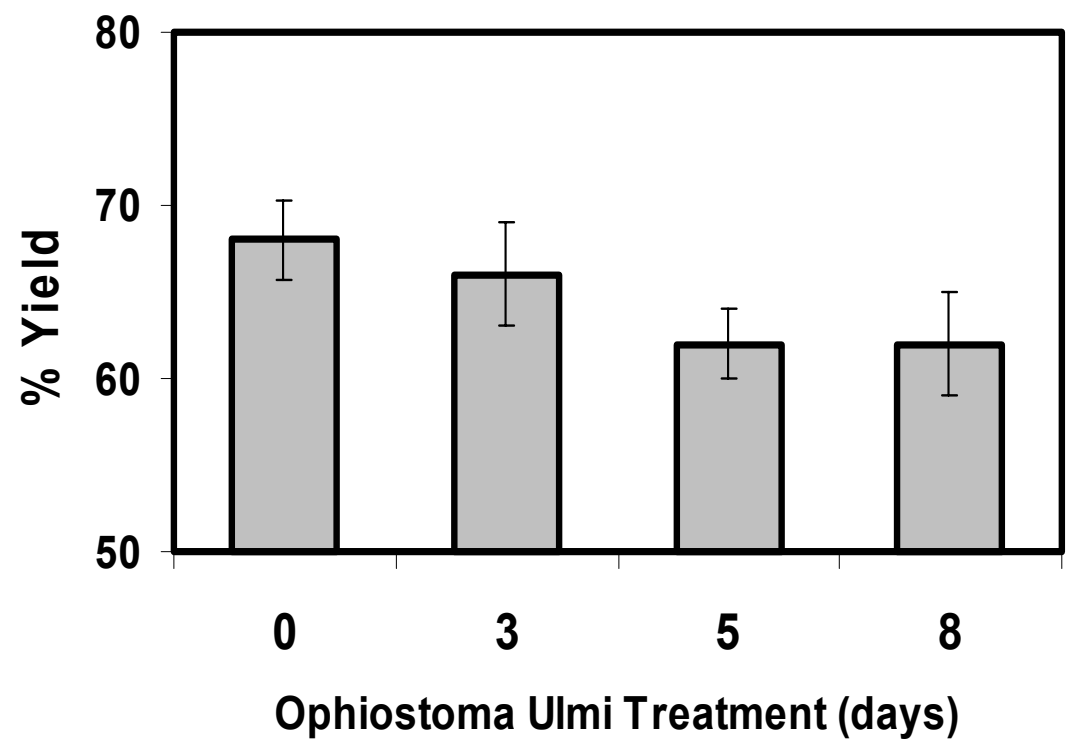

Fig.1. Yield of cellulose microfibrils with different Ophiostoma Ulmi treatment conditions

The low cellulolytic activity of OS1 fungus was further confirmed by a study of the weight loss and cellulose content of the treated fibres. The loss in fibre weight, as depicted in Fig. 2, showed a gradual drop up to a maximum $7.5 \%$ of original fibre weight for 4 days treatment and tended to be insignificant thereafter. A similar trend is seen with respect to the cellulose content of the treated fibres. This is evident in Fig. 3, where the cellulose content is seen to decrease with the extent of treatment, and the loss of cellulose is proportionate with the weight loss of the treated fibres.

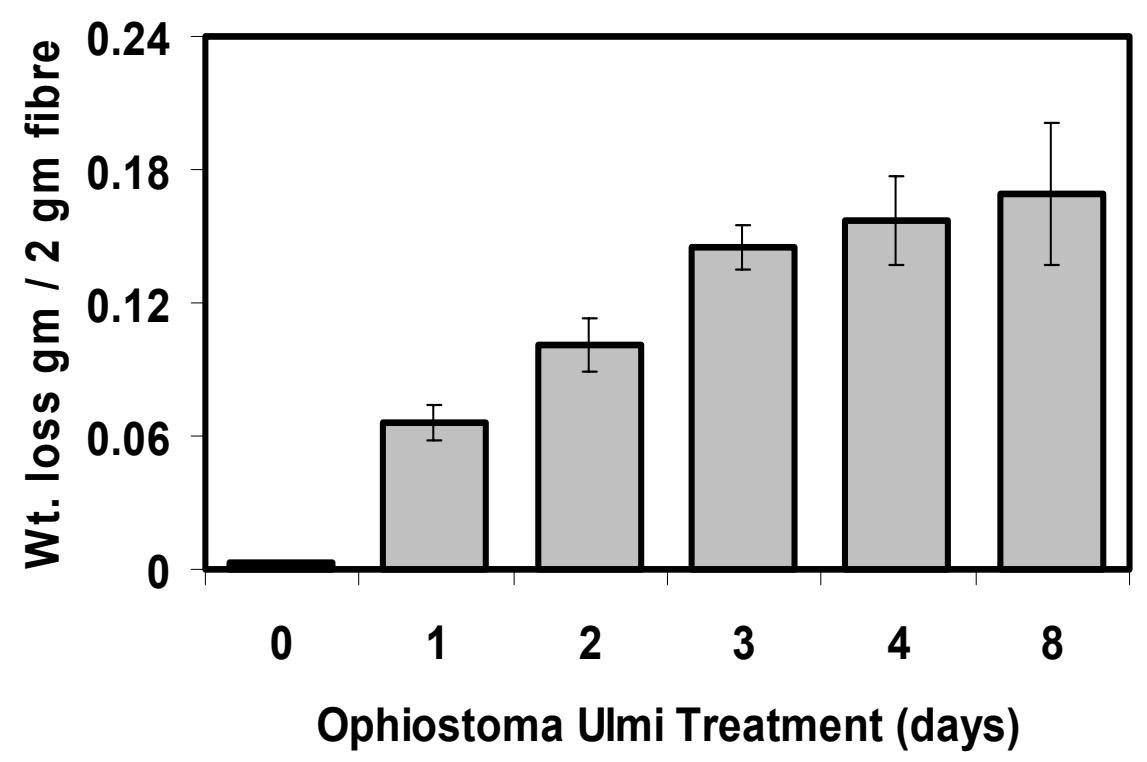

Fig. 2. Weight loss of fibres with different OS1 fungus treatment conditions 


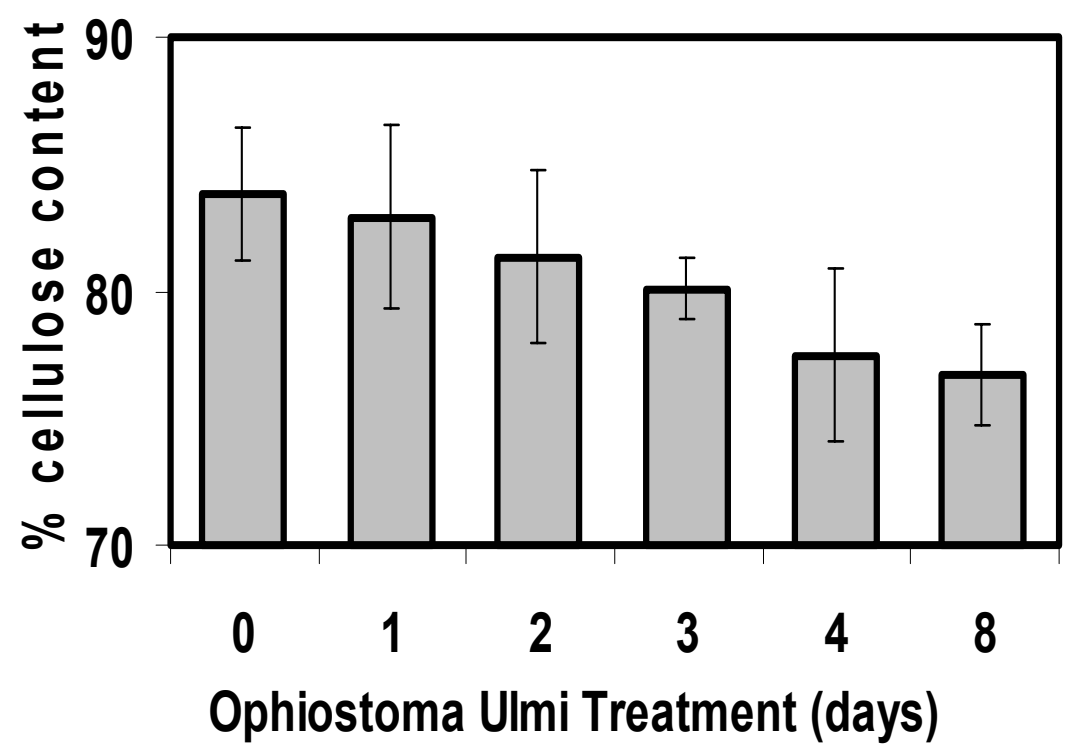

Fig. 3. Cellulose content of fibres with different OS1 treatment conditions

The weight loss and a proportionate decrease in cellulose content of the treated fibres imply that the action of fungal enzymes on the fibres is mostly limited to cellulose and not the hemicellulose. Now, the reason for this low level of activity against cellulose can be explained only once the specific enzymes are isolated and identified. This is the next phase of this project.

\section{Microfibril Diameter Distribution}

Having understood the level of OS1 activity against cellulose, it is vital to understand the effect of OS1 fungal treatment on the internal defibrillation tendency of the treated fibres during subsequent mechanical defibrillation techniques such as high shear refining or high-pressure homogenization. This is the first step towards testing the hypothesis that enzymes can help in internal defibrillation through either weakening the hydrogen bonds that exist between microfibrils or loosening up the fibrils through controlled hydrolytic activity.

Fibres treated with OS1 fungus were refined and the number-average diameter distributions of these refined fibres for a 4 days treatment are detailed in Fig. 4. A very significant shift in the diameter distribution of the fibres occurred towards the lower diameter range, with the maximum yield of fibres below $100 \mathrm{~nm}$ range for the 4 days treated fibres, while that for the untreated fibre were between $100-250 \mathrm{~nm}$ range. The fibre diameter distribution did not change in an appreciable manner with increase in treatment time longer than 4 days treatment (results not shown here). This shift in fibre diameter distribution curve towards the lower diameter range for a treated fibre after the refining is of importance in this work as this observed phenomenon can happen only if the treatment had an effect of facilitating the internal defibrillation in the fibre during refining. The mechanism is not apparent yet, but a good supposition is that the enzymes might have worked to reduce the hydrogen bonding between the fibrils, thus improving 
the internal defibrillation during refining. This concept is more visible in the TEM images of an unrefined treated fibre as shown in Fig. 5 and the refined fibres treated with the fungus as detailed in Fig. 6.

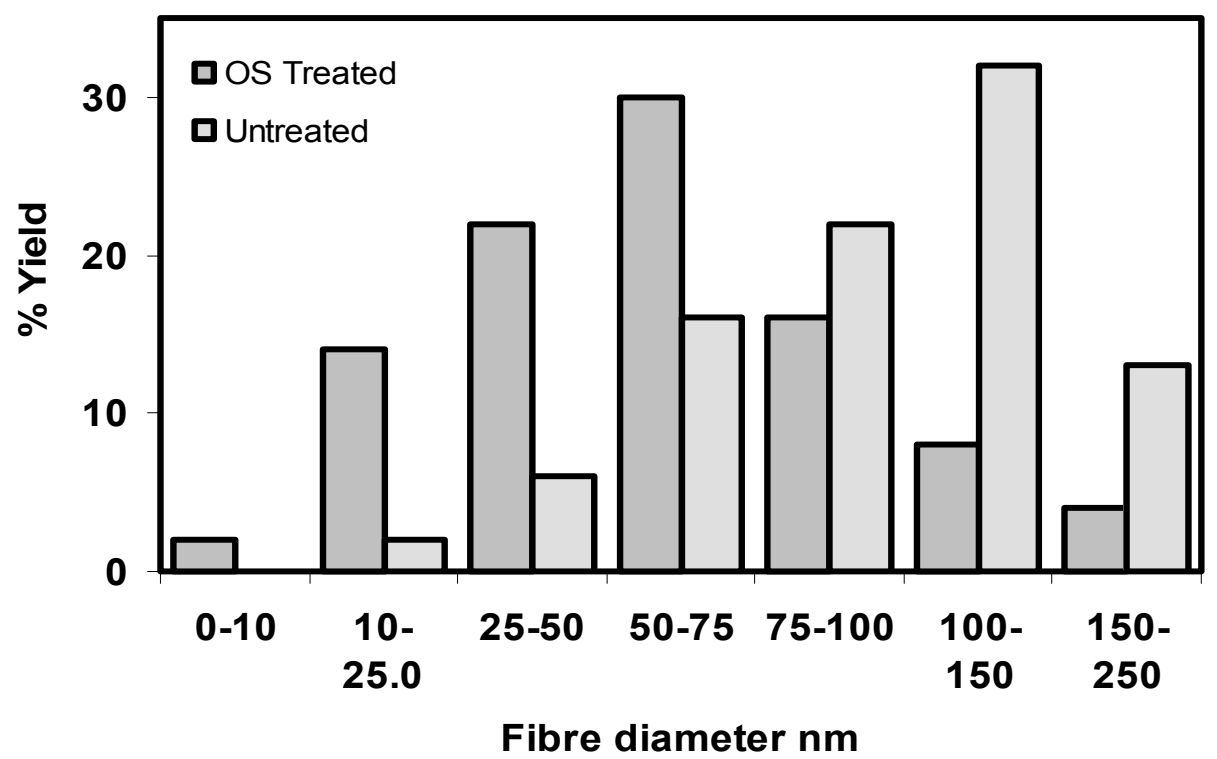

Fig. 4. Effect of OS1 fungal treatments on number averaged diameter distribution of fibres after refining for a 4 days treatment

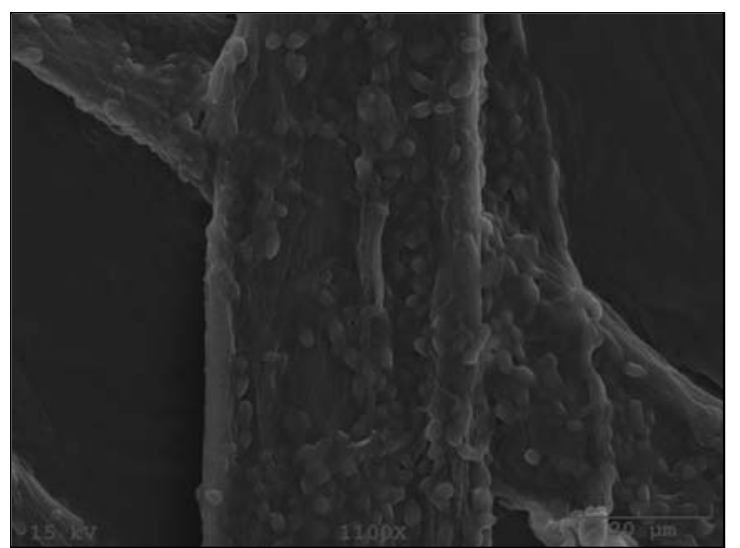

(a)

Fig. 5. OS1 fungal treatment of fibres, (a) fungus growing on the fibre, (b) treated fibre before high shear refining

The fibrillation of the treated fibres, as seen in Fig.6 (b), is more pronounced after refining, compared to the untreated fibres. The actual separation of elementary fibres takes place to a good extent with treated fibres, while high shear refining seems to have a reduced fibril separation effect on untreated fibres. This observation can explain the difference in fibre diameter distribution associated with treated and untreated fibres.

Janardhnan and Sain (2006). "Cellulose Microfibril Isolation," BioResources 1(2), 176-188. 183 


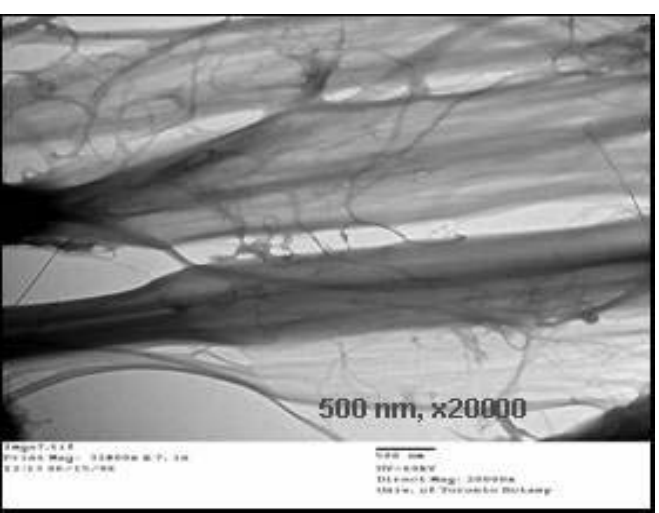

(a)

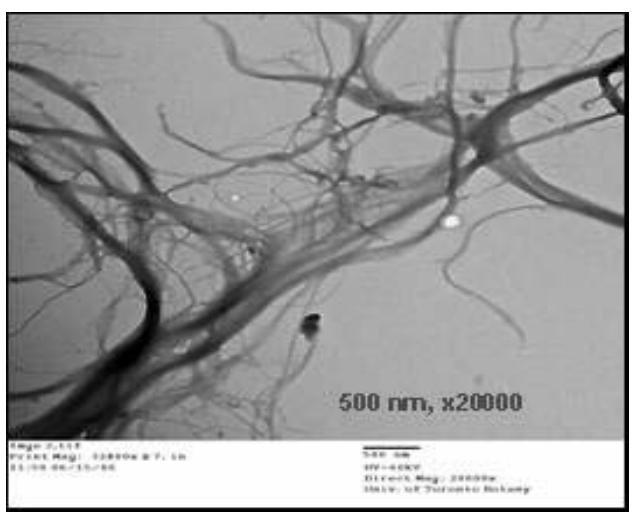

(b)

Fig. 6. Effect of OS1 fibre treatments on internal defibrillation - (a) TEM of untreated fibre after high shear refining, (b) TEM of treated fibre after high shear refining

Cryocrushing is the final step that helps in the isolation of cellulose microfibrils into individual entities from the fibrillated fibres. An interesting point to note here is that a significant difference in fibre diameter distribution observed between treated and untreated fibres after refining, as was detailed in Fig. 4, no longer seems to demonstrate their significance in Fig. 7, which depicts the fibre diameter distribution of treated and untreated fibres after cryocrushing. The reason for such a distribution may be explained by a strong and positive effect of cryocrushing on microfibril isolation from fibres, such that the better defibrillation attained by treated fibres after refining is subdued.

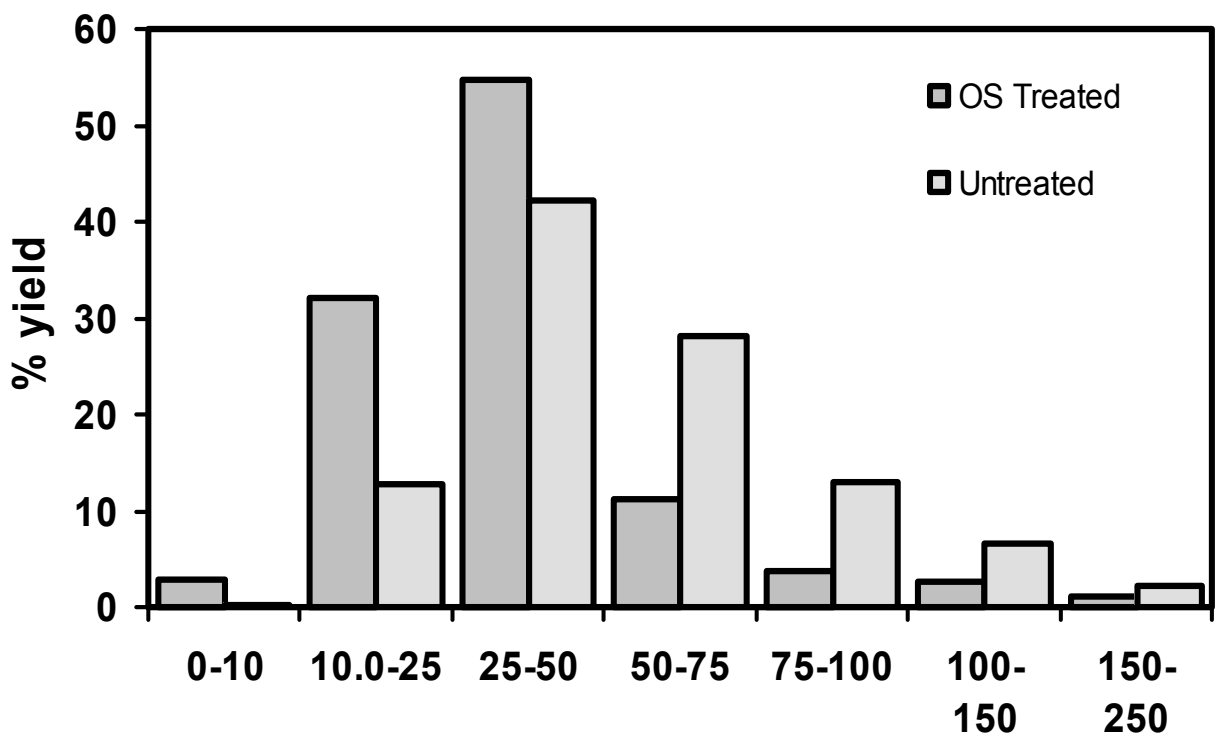

Fibre diameter $\mathrm{nm}$

Fig.7. Effect of OS1 treatments of fibres on the yield and distribution of cellulose microfibrils after refining and cryocrushing 
The fibre diameter distribution trend is similar for both treated and untreated fibres, with microfibrils from treated fibres showing a slight shift towards lower diameter range, and with the major fraction of fibres in the $0-50 \mathrm{~nm}$ range. The cellulose microfibrils isolated from treated fibres after cryocrushing showed very clear and distinct separation, as compared to cellulose microfibrils isolated from untreated fibre, as seen in Fig. 8 and Fig. 9.

This narrow shift in the fibre diameter distribution, as shown in Fig. 8, stems from the fact that isolation of cellulose microfibrils into distinct entities is not as good with untreated fibres as with treated fibres. This effect is evident from a closer look at TEM pictures, as shown in Fig. 8 and Fig. 9.

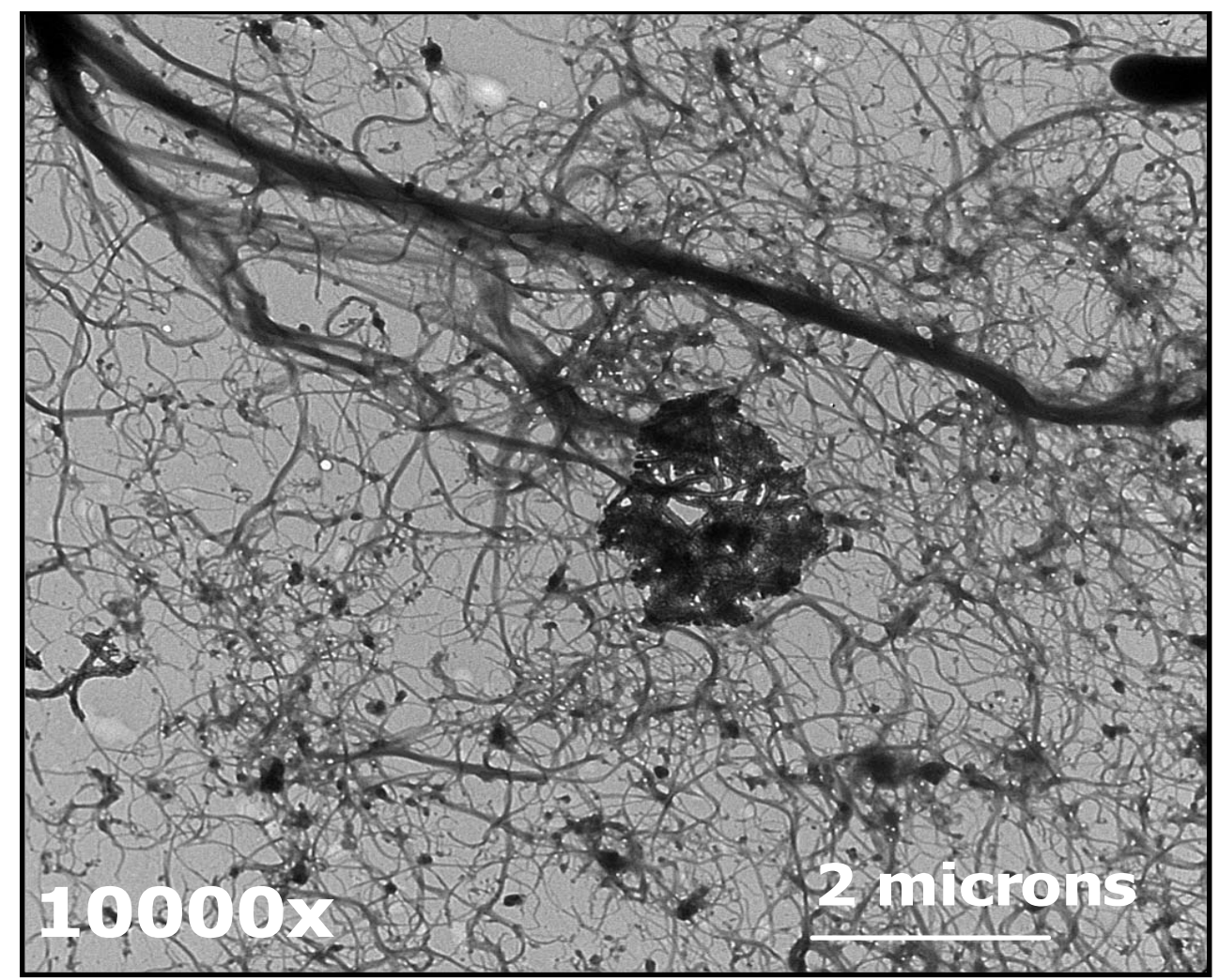

Fig. 8. TEM of cellulose microfibrils isolated from OS1 treated fibres through refining and cryocrushing 


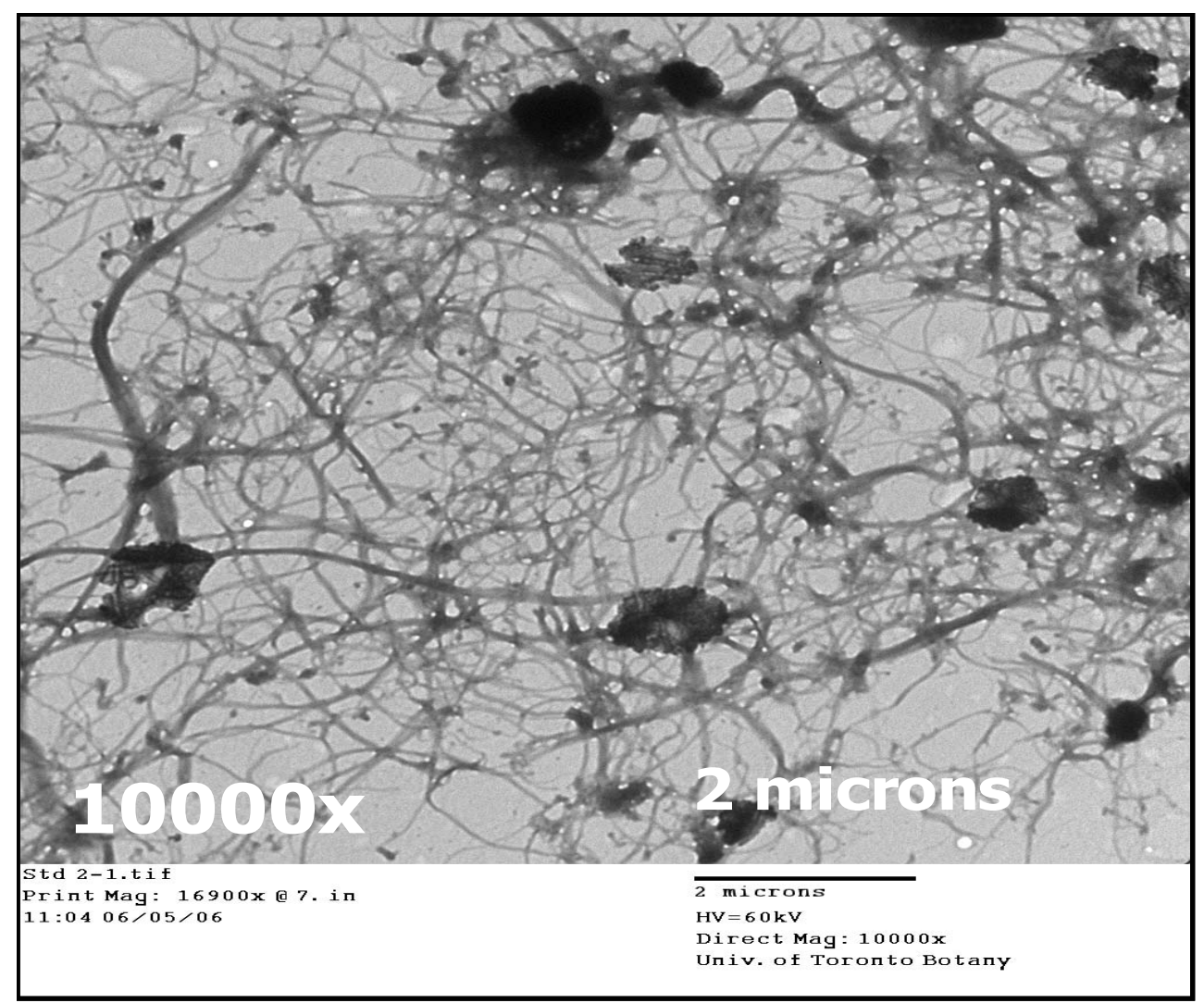

Fig. 9. TEM of cellulose microfibrils isolated from untreated fibres through refining and cryocrushing

The observations detailed above steer our thinking in two directions.

(a). The effect of fungal treatment has been shown to have a significant impact on the defibrillation characteristics of the fibres during defibrillation techniques such as the PFI refining we have used here. However, the impact seems to lose its significance once these refined fibres are cryocrushed. Therefore one may ask whether this enzymatic fibre treatment really benefits the isolation of cellulose microfibrils in a two-step process that includes PFI refining and cryocrushing.

(b). Having demonstrated the encouraging effect of OS1 treatment on the defibrillation of fibres during subsequent refining, it is worthwhile adopting a one-step process such as homogenization in a microfluidizer to authenticate the effect of fibre treatment and see if fewer passes through the microfluidizer are enough to isolate cellulose microfibrils, as compared to the number of passes required for untreated fibres.

In addition to isolating and identifying the extracellular enzymes involved in the treatment, the next phase of this work will include the isolation of cellulose microfibrils from Ophiostoma-treated fibre, using single-step / multipass high-pressure homogeniz- 
ation. Fewer passes for treated fibres through a homogenizer for comparable microfibril yield will undeniably suggest a lower energy scenario in cellulose microfibril isolation.

\section{CONCLUSIONS}

1. The fungus OS1 treatment was shown to have a significant impact on the defibrillation characteristics of the fibres - a major step in the isolation of cellulose microfibrils.

2. Cellulose microfibrils isolated by refining and cryocrushing of treated fibres yielded very distinct microfibrils and a narrower microfibril diameter distribution, compared to that obtained for untreated fibres.

3. The fungus OS1 treatment of bleached kraft fibres seems to have only a mild activity against cellulose, which is of interest to this work, as this minimizes the loss of cellulose.

\section{ACKNOWLEDGMENTS}

The authors are grateful for the support of Natural Science and Engineering Research Council of Canada - BIOCAP.

\section{REFERENCES CITED}

Bolaski, W., Gallatin, A., and Gallatin, J. C. (1959). "Enzymatic conversion of cellulosic fibers," United States Patent 3,041,246.

Chakraborty, A., Sain, M., and Kortschot, M. (2005). "Cellulose microfibres: A novel method of preparation using high shear refining and cryocrushing," Holzforschung 60(1), 53-58.

Fengel, D., and Wegener, G. (1984). "Wood: chemistry, ultra structure, reactions," W. de Gruynter, New York.

Fuentes, J. L., and Robert, M. (1988). "Process of treatment of a paper pulp by an enzymic solution," European Patent 262040.

Herrick, F. W., Casebeir, R. L., Hamilton, J. K., and Sandberg, K. R. (1983). J. Appl. Polymer. Sci.: Appl. Polymer Symp. 37, 797-813.

Jurasek, L., and Paice, M. G. (1988). "Biological treatment of pulps," Biomass 15, 103108.

Liang, C. Y., and Marchessault, R. H. (1959). "Infrared spectra of crystalline polysaccharides. I. Hydrogen bonds in native celluloses ," J. Polym. Sci. 37, 385395.

Morvan, C., Jauneau, A., Flaman, A., Millet, J, and M. Demarty. (1990). "Degradation of flax polysaccharides with purified endo-polygalacturonidase," Carbohyd. Polym. 13, 149-163.

Nazareth, S., and Mavinkurve, S. (1987). "Laboratory studies on retting of coconut husk," Int. Biodeter. 23, 343-355. 
Nissan, A. H., Byrd, V. L., Batten, G. L., Jr., and Ogden, R. W. (1985). "Paper as an Hbond dominated solid in the elastic and plastic regimes," Tappi J. 68(9), 118-122.

Nomura, Y. (1985). "Digestion of pulp," Japanese Patent 126,395/85.

Paice, M. G., and Jurasek, L. (1984). "Removing hemicellulose from pulps by specific enzymic hydrolysis," J. Wood Chem. Technol. 4(2), 187-198.

Senior, D. J., Mayers, P. R., Miller, D., Sutcliffe, R., Tan, L. and Saddler, J. N. (1988). "Selective solubilization of xylan in pulp using a purified xylanase from Trichoderma harzianum," Biotechnol. Lett. 10, 907-912.

Sharma, H. S. S. (1987). "Screening of polysaccharide-degrading enzymes for retting flax stems," Int. Biodeter. 23,181-186.

Tashiro, K., and Kobayashi, M. (1991). "Theoretical evaluation of three-dimensional elastic constants of native and regenerated celluloses: role of hydrogen bonds," Polymer 32, 1516.

Tolan, J. S., and Canovas, R.V. (1992). "The use of enzymes to decrease the C12 requirements in pulp bleaching," Pulp Paper Can. 93, 39-42.

Uchimoto, I., Endo, K., and Yamagishi, Y. (1988). "Improvement of deciduous tree pulp," Japanese Patent. 135,597/88.

Viikari, L., Kantelinen, A. Poutanen, and Ranua, M. (1990). "Characterization of pulps treated with hemicellulolytic enzymes prior to bleaching," In: Kirk, T. K. and Chang, H.-m. (Eds.) Biotechnology in Pulp and Paper Manufacture, ButterworthHeinemann, Boston, pp. 145-151.

Yerkes, W. D. (1968). "Process for the digestion of cellulosic materials by enzymatic action of Trametes suaveolens," United States Patent 3,406,089.

Zobel, B. J. and McElwee, R. L. (1966). "Variation of cellulose in loblolly pine" Tappi J. 49(9), 383-387

Article submitted August 16, 2006; Revision accepted September 20, 2006; Published September 21, 2006 